\title{
Correlation of caval index, inferior vena cava diameter, and central venous pressure in shock patients in the emergency room
}

This article was published in the following Dove Press journal:

Open Access Emergency Medicine

19 August 2014

Number of times this article has been viewed

\author{
Panita Worapratya' \\ Sawanya Anupat ${ }^{2}$ \\ Ruedeekorn Suwannanon ${ }^{3}$ \\ Prasit Wuthisuthimethawee' \\ 'Department of Emergency Medicine, \\ Songklanagarind Hospital, Faculty \\ of Medicine, Prince of Songkla \\ University, Songkhla, Thailand; \\ 'Emergency Department, Bangkok \\ Samui Hopsital, Suratthanee, \\ Thailand; ${ }^{3}$ Department of Radiology, \\ Songklanagarind Hospital, Faculty of \\ Medicine, Prince of Songkla University, \\ Songkhla, Thailand
}

Objectives: This study aims to determine the correlation of the caval index, inferior vena cava (IVC) diameter, and central venous pressure (CVP) in patients with shock in the emergency room.

Materials and methods: This is a prospective double-blind observational study conducted in the emergency room of a tertiary care center. All patients who presented with shock and had a central venous catheter insertion performed were enrolled. The caval index was calculated as a relative decrease in the IVC diameter during the normal respiratory cycle. The correlation of CVP and the caval index were calculated by Pearson's product-moment correlation coefficient.

Results: Among the 30 patients enrolled, the median age was $59.90 \pm 21.81$ years and $17(56.7 \%)$ patients were men. The summary statistics that were generated for the participants' characteristics were divided into CVP $<10 \mathrm{~cm} \mathrm{H}_{2} \mathrm{O}, 10-15 \mathrm{~cm} \mathrm{H}_{2} \mathrm{O}$, and $>15 \mathrm{~cm} \mathrm{H}_{2} \mathrm{O}$. The correlation of the CVP measurement with the ultrasound IVC caval index was $r=-0.721(P=0.000)$ by two-dimensional mode ultrasound and $r=-0.647(P=0.001)$ by M-mode. The correlations of CVP with the end-expiratory IVC diameter were $r=0.551(P=0.002)$ by two-dimensional mode ultrasound and $r=0.492(P=0.008)$ by M-mode. The sensitivity and specificity of the caval index were calculated to predict the CVP. The results showed that the cut-off points of the caval index were 30, 20, and 10 at CVP levels $<10 \mathrm{~cm} \mathrm{H}_{2} \mathrm{O}, 10-15 \mathrm{~cm} \mathrm{H}_{2} \mathrm{O}$, and $>15 \mathrm{~cm} \mathrm{H}_{2} \mathrm{O}$, respectively.

Conclusion: The caval index calculated from the IVC diameter measured by bedside ultrasound in the emergency room has a good correlation with CVP.

Keywords: hemodynamic evaluation, inferior vena cava diameter, caval index

\section{Introduction}

\section{Background}

Shock is a common problem in the emergency room. Optimized fluid resuscitation in the emergency room is challenging and crucial in shock patients. Since protocols for early goal-directed therapy in severe sepsis and septic shock were first published and became standardized, central venous access appears to be important for fluid evaluation and serves as a guide for fluid resuscitation. However, invasive procedures such as central venous catheter insertion have many complications and require substantial amounts of time.

Songklanagarind Hospital (Songkhla, Thailand) is a medical training and tertiary care center in southern Thailand. Annually, there are 1,500 cases of shock patients in the emergency room who require central venous catheter insertion, which accounts for as many as one-third of the emergency visits each year. The complication rates
Department of Emergency Medicine, Songklanagarind Hospital, Faculty of Medicine, Prince of Songkla University, Hat Yai, Songkhla 90I I0, Thailand

Tel $+667445 \quad 1705$

Fax $+667445 \quad 1704$

Email pikkokung@yahoo.com 
of central venous catheter insertion, such as infection, hematoma, pneumothorax, and bleeding, depend upon the operator's experience. ${ }^{1-4}$

Bedside ultrasonography is a noninvasive technique and is readily available in the Songklanagarind Hospital emergency room. Using bedside ultrasonography in conjunction with clinical parameters (for example, vital signs and urine output) can be useful in determining the volume status in shock patients. The inferior vena cava (IVC) is a large vein that can be easily identified by transabdominal ultrasonography when the orientation probe is at the subxiphoid of patients lying in the supine position. Collapsibility of the IVC diameter, termed the caval index, has been documented as being correlated with right atrium pressure among selected patients in a nonacute care setting, ${ }^{5-7}$ and three research papers have documented the IVC diameter as an index of right-sided cardiac function. ${ }^{8,9}$ Many research papers use collapsibility of the IVC as an initial tool for early detection of hypovolemic shock ${ }^{10}$ or as an indicator of acute blood or volume loss. ${ }^{11-13}$ In 2007 , Brennan et $a 1^{6,7}$ used the IVC for right atrium pressure and found that an IVC diameter of $2 \mathrm{~cm}$ has good correlation with a right atrium pressure of $10 \mathrm{mmHg}$, with a sensitivity of $73 \%$ and specificity of $85 \%$. In addition, $40 \%$ collapsibility of the IVC has been correlated with a sensitivity of $40 \%$ and specificity of $84 \% .{ }^{13}$ Therefore, in 2010, guidelines were published for the echocardiographic assessment of the right heart in adults. ${ }^{14}$ Nowadays, using the IVC diameter to predict hemodynamic status has been extended into acute care settings, such as in ventilated septic patients in the intensive care unit ${ }^{15}$ and in the emergency room. ${ }^{16}$

However, there are not enough data using the IVC diameter and collapsibility to determine volume status in the emergency room, especially in Thailand. This study aims to determine the correlation of the caval index, IVC diameter, and central venous pressure (CVP) in patients with any cause of shock in the emergency room.

\section{Importance}

Bedside ultrasonography can be a useful tool for the initial evaluation of the volume status of shock patients in an emergency room setting as an adjunct to clinical data.

\section{Materials and methods}

\section{Study design and setting}

This is a prospective, observational study that was conducted in the emergency room of a medical tertiary care center in southern Thailand where there are more than 40,000 visitors a year.

\section{Selection of participants}

Candidates for this study were shock patients who presented in the emergency room of Songklanagarind Hospital between September 1, 2012 and November 15, 2012. Inclusion criteria were age $>18$ years and the requirement of a central venous catheter. The exclusion criteria were pregnancy, postcardiac arrest, intraabdominal compartment syndrome, ventilated patients, and any patient who was not suitable for bedside ultrasonography, which was to measure the IVC, such as among morbidly obese patients or when ultrasonography was limited by dilated bowel loops. All candidates gave consent before the procedure began, if they were conscious. For patients with an altered mental status, consent was obtained from each patient's health care proxy.

\section{Methods of measurement and outcome measures}

The patients needed to be in the supine position for IVC measurement, which took place at $3 \mathrm{~cm}$ from the right atrial border during normal full inspiratory and expiratory phases. Third-year residents in emergency medicine training captured the pictures and made video recordings during the procedure. After that, another emergency physician and radiologist reviewed the pictures and video records and measured the maximal and minimal diameters of the IVC in two-dimensional (2D)-mode and M-mode. CVPs were recorded immediately by the nurses at the time of bedside ultrasonography. The three operators - the nurse, the emergency physician who performed the bedside ultrasonography, and the radiologist who measured the IVC diameter - were blinded to the data obtained by each other. Everyone who performed the bedside ultrasonography attended an ultrasonography class for emergency physicians, and they also had competency to perform ultrasonography at the bedside.

\section{Primary data analysis}

Summary statistics were performed on patient demographics (ie, age and sex) and the patients' clinical characteristics (ie, vital signs, serum lactate level, expiratory IVC diameter, and caval index). The patients were divided into one of three groups by CVP $<10 \mathrm{~cm} \mathrm{H}_{2} \mathrm{O}, 10-15 \mathrm{~cm} \mathrm{H}_{2} \mathrm{O}$, and $>15 \mathrm{~cm} \mathrm{H}_{2} \mathrm{O}$. Their characteristics, vital signs, lactate levels, and primary study measurements were reported in terms of mean values and percentages. The analysis of variance test and Fisher's exact test were used for comparisons 
Table I Demographic data of 30 patients

\begin{tabular}{|c|c|c|c|c|}
\hline \multirow[t]{2}{*}{ Demographic data } & \multicolumn{3}{|c|}{ Central venous pressure $\left(\mathrm{cm} \mathrm{H}_{2} \mathrm{O}\right)$} & \multirow[t]{2}{*}{$P$-value } \\
\hline & $<10$ & $10-15$ & $>15$ & \\
\hline Number of patients & 7 & 6 & 17 & \\
\hline $\operatorname{Sex}(\%)$ & & & & 1.000 \\
\hline Male & $4(57.1)$ & $3(50.0)$ & $10(58.8)$ & \\
\hline Female & $3(42.9)$ & $3(50.0)$ & $7(4 \mid .2)$ & \\
\hline Average age, years (SD) & $49.1(24.3)$ & $74.5(10.0)$ & $59.2(21.9)$ & 0.108 \\
\hline Average BMI, kg/m² (SD) & $22.0(2.1)$ & $26.2(4.4)$ & $23.8(2.8)$ & 0.065 \\
\hline Underlying disease (\%) & & & & 1.000 \\
\hline At least one & $5(7 \mid .4)$ & $4(66.7)$ & $13(76.5)$ & \\
\hline Unknown & $2(28.6)$ & $2(33.3)$ & $4(23.5)$ & \\
\hline Central venous insertion (\%) & & & & 0.084 \\
\hline Internal jugular vein & $4(57.1)$ & $6(100)$ & I5 (88.2) & \\
\hline Subclavian vein & $3(42.9)$ & $0(0)$ & $2(11.8)$ & \\
\hline \multicolumn{5}{|l|}{ Mean vital signs (SD) } \\
\hline Pulse rate (beat/min) & II $3.4(32.9)$ & $88.7(10.2)$ & $100.8(21.7)$ & 0.178 \\
\hline Respiratory rate (breath/min) & $28.1(4.0)$ & $27.0(3.7)$ & $28.0(9.1)$ & 0.953 \\
\hline Mean arterial pressure & $65.0(13.5)$ & $60.9(7.7)$ & $60.3(10.0)$ & 0.597 \\
\hline Serum lactate (mg/dL) & $4.3(4.0)$ & $1.9(1.7)$ & $4.1(4.0)$ & 0.715 \\
\hline Oxygen saturation (\%) & $98.6(1.8)$ & $98.2(1.2)$ & $87.2(20.7)$ & 0.244 \\
\hline
\end{tabular}

Abbreviations: SD, standard deviation; BMI, body mass index.

between the groups. Pearson's product-moment correlation coefficients were used to analyze the correlation coefficients: $r$ of CVP; end-expiratory IVC (eIVC) diameter; and caval index.

\section{Secondary data analysis}

The correlation of the IVC diameter and CVP was reported as sensitivity and specificity.

\section{Results}

\section{Primary outcome}

There were 69 patients who presented with shock in the emergency room during the study period; only 30 patients matched the inclusion criteria. Twenty-one patients were excluded due to their response to the initial volume resuscitation, as a central line was not needed; 12 patients were unable to provide informed consent or they did not sign consent forms; and in six patients, it was hard to identify the IVC because of thick abdominal walls.

Among the 30 patients, there were various types of shock including sepsis, hypovolemic, and cardiogenic shock. The route of venous catheter insertion was chosen according to the medical caregiver's experience. After dividing 30 patients into one of three groups, there were no differences in CVP measurements by age, sex, and type of shock (Table 1).

The correlations of the CVP measurement with the ultrasound IVC caval index were $r=-0.721(P=0.000)$ by $2 \mathrm{D}$-mode ultrasound and $r=-0.647(P=0.001)$ by M-mode. The
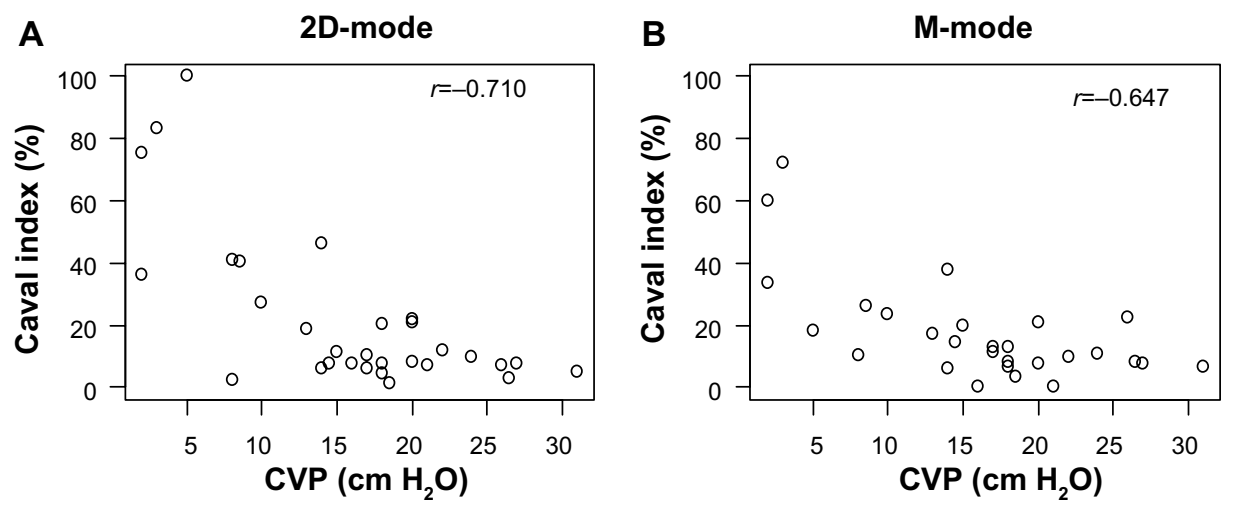

Figure I Correlation between CVP and the caval index using 2D-mode and M-mode bedside ultrasonography in patients with altered mental status. Notes: (A) 2D-mode; (B) M-mode.

Abbreviations: 2D, two-dimensional; CVP, central venous pressure. 

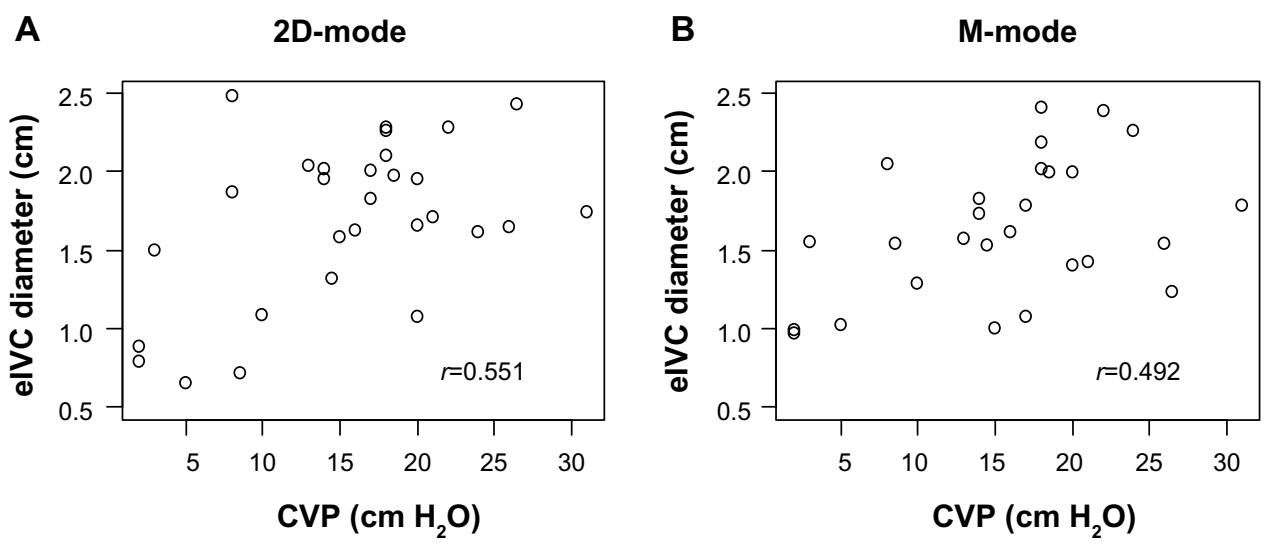

Figure 2 Correlation of elVC diameter and caval index in patients who could follow commands.

Notes: (A) 2D-mode; (B) M-mode.

Abbreviations: 2D, two-dimensional; elVC, end-expiratory inferior vena cava; CVP, central venous pressure.

correlations of CVP with the eIVC diameter were $r=0.551$ $(P=0.002)$ by $2 \mathrm{D}$-mode ultrasound and $r=0.492(P=0.008)$ by M-mode. The sensitivity and specificity of the caval index were calculated to predict the CVP. The results showed that the cut-off points of the caval index were 30,20, and 10 at CVP levels of $<10 \mathrm{~cm} \mathrm{H}_{2} \mathrm{O}, 10-15 \mathrm{H}_{2} \mathrm{O}$, and $>15 \mathrm{~cm} \mathrm{H}_{2} \mathrm{O}$, respectively (Figures 1 and 2).

\section{Secondary outcomes}

We divided the patients into one of three groups: CVP $<10 \mathrm{~cm} \mathrm{H}_{2} \mathrm{O} ; 10-15 \mathrm{~cm} \mathrm{H}_{2} \mathrm{O}$; and $>15 \mathrm{~cm} \mathrm{H}_{2} \mathrm{O}$. The sensitivity and specificity of the caval index at the cutoff point of each group are shown in Table 2-4.

\section{Conclusion}

Of the 30 patients with various causes of shock, there was a correlation between the caval index and the IVC diameter. A CVP of $<10 \mathrm{~cm} \mathrm{H}_{2} \mathrm{O}$ correlated with a caval index of 30 (sensitivity 85.7 , specificity 95.7 ), a CVP of $10-15 \mathrm{~cm} \mathrm{H}_{2} \mathrm{O}$ correlated with a caval index of 20 (sensitivity 33.3 , specificity 62.5 ), and a CVP of $>15 \mathrm{~cm} \mathrm{H}_{2} \mathrm{O}$ correlated with a caval index of 10 (sensitivity 76.5 , specificity 76.9 ).

\section{Limitations}

The bedside ultrasonographic measurements of the IVC were done by third-year emergency residents who had the

Table 2 Sensitivity and specificity of the caval index in Group I, central venous pressure $<10 \mathrm{~cm} \mathrm{H}_{2} \mathrm{O}$

\begin{tabular}{lll}
\hline Caval index & Sensitivity (\%) & Specificity (\%) \\
\hline 20 & 85.7 & 78.3 \\
30 & 85.7 & 95.7 \\
40 & 71.4 & 95.7 \\
50 & 42.9 & 100 \\
80 & 28.6 & 100 \\
\hline
\end{tabular}

competency to perform bedside ultrasonography, and the video recordings of this procedure were sent to a radiologist to record and interpret the diameter of the IVC and caval index. Everyone who performed bedside ultrasonography in this study was trained in a demonstration room for 3 hours. However, each patient had bedside ultrasonography performed by a single resident without repeating the procedure by another physician. Therefore, interobserver reliability was not measured.

The timing of bedside ultrasonography after central line insertion, as well as the amount of volume loading, were not recorded. However, ultrasonography was performed immediately after successful central venous catheter insertion; recording of the CVP was also performed. Therefore, there were no time differences between central line insertion and bedside ultrasonography.

This study could not perform the eIVC diameter measurement in some patients because of dyspnea or altered mental status. Therefore, we performed the caval index and end-expiratory caval index - both of which correlated well with the CVP.

Finally, even though shock is a common presentation in the emergency room, we only had 30 patients in this study. It was not possible to receive informed consent from some patients (such as those with altered mental status), and sometimes

Table 3 Sensitivity and specificity of the caval index in Group 2, central venous pressure $10-15 \mathrm{~cm} \mathrm{H}_{2} \mathrm{O}$

\begin{tabular}{lll}
\hline Caval index & Sensitivity (\%) & Specificity (\%) \\
\hline 20 & 33.3 & 62.5 \\
30 & 16.7 & 75.0 \\
40 & 16.7 & 79.2 \\
50 & 0 & 87.5 \\
\hline
\end{tabular}


Table 4 Sensitivity and specificity of the caval index in Group 3, central venous pressure $>15 \mathrm{~cm} \mathrm{H}_{2} \mathrm{O}$

\begin{tabular}{lll}
\hline Caval index & Sensitivity (\%) & Specificity (\%) \\
\hline 10 & 76.5 & 76.9 \\
20 & 82.4 & 61.5 \\
30 & 100 & 53.8 \\
40 & 100 & 46.2 \\
\hline
\end{tabular}

third-year residents who had competency to perform bedside ultrasonography were not available. Moreover, in six patients, the IVC diameter could not be identified, which is a normal limitation of most bedside ultrasonography. As in several previous studies, an IVC measurement was not possible in $10 \%-15 \%$ of patients because of a thick abdominal wall and excessive intraluminal bowel gas. ${ }^{17,18}$

\section{Discussion}

The primary endpoint of this study was to examine the association between the diameter of the IVC and CVP. In this study, we performed the caval index and end-expiratory caval index. Both the caval index and endexpiratory caval index had statistical significant correlations with the CVP ( $r=-0.710[P=0.000]$ and $r=0.492[P=0.008]$, respectively). These data corresponded well with the results of Schefold et al. ${ }^{15}$

For the secondary endpoints of this study, we divided the caval index into one of three groups: $\mathrm{CVP}<10 \mathrm{~cm} \mathrm{H}_{2} \mathrm{O}$; $10-15 \mathrm{~cm} \mathrm{H}_{2} \mathrm{O}$; and $>15 \mathrm{~cm} \mathrm{H}_{2} \mathrm{O}$. We then examined the appropriate cut-off point of each caval index and CVP measurement. Our appropriate cut-off points of the caval index for CVPs $<10 \mathrm{~cm} \mathrm{H}_{2} \mathrm{O}, 10-15 \mathrm{~cm} \mathrm{H}_{2} \mathrm{O}$, and $>15 \mathrm{~cm} \mathrm{H}_{2} \mathrm{O}$ were 30,20 , and 10, respectively. These were lower than those noted in the study by Rudski et al, ${ }^{14}$ because the authors used the end-expiratory caval index instead of the caval index, which we used in this study. We examined the correlation between the CVP and caval index to determine which is more feasible, rather than using the end-expiratory caval index, because many patients in the emergency room were in shock and could not follow the commands necessary for an end-expiratory measurement. The sensitivity and specificity of each cut-off point in determining the CVP of this study were not high because of interobserver variability. However, we tried to minimize interobserver variability by choosing third-year emergency medicine residents who took a 3-hour demonstration class for IVC measurement.

The amount of volume loading in the time between CVP and bedside ultrasonography caval index measurements was not recorded because we kept the vein open while the CVP measurement and bedside ultrasonography were performed at the same time.

In this study, the nurses who measured the CVP, the third-year residents in emergency training who performed the bedside ultrasonography, and the radiologist who reported the caval index were blinded to the data obtained by each other.

\section{Disclosure}

The authors report no conflicts of interest in this work.

\section{References}

1. Ziai WC, Chandolu S, Geocadin RG. Cerebral herniation associated with central venous catheter insertion: risk assessment. J Crit Care. 2013;28(2):189-195.

2. Kusminsky RE. Complications of central venous catheterization. JAm Coll Surg. 2007;204(4):681-696.

3. Bishop L, Dougherty L, Bodenham A, et al. Guidelines on the insertion and management of central venous access devices in adults. Int J Lab Hematol. 2007;29(4):261-278.

4. Yamanaka T, Miyazaki Y, Sato M. [Retrograde cavernous sinus air embolism after central venous catheter removal]. No Shinkei Geka. 2012;40(11):991-995. Japanese.

5. Kircher BJ, Himelman RB, Schiller NB. Noninvasive estimation of right atrial pressure from the inspiratory collapse of the inferior vena cava. Am J Cardiol. 1990;66(4):493-496.

6. Brennan JM, Blair JE, Goonewardena S, et al. A comparison by medicine residents of physical examination versus hand-carried ultrasound for estimation of right atrial pressure. Am J Cardiol. 2007;99(11): 1614-1616.

7. Brennan JM, Ronan A, Goonewardena S, et al. Handcarried ultrasound measurement of the inferior vena cava for assessment of intravascular volume status in the outpatient hemodialysis clinic. Clin J Am Soc Nephrol. 2006;1(4):749-753.

8. Moreno FL, Hagan AD, Holmen JR, Pryor TA, Strickland RD, Castle $\mathrm{CH}$. Evaluation of size and dynamics of the inferior vena cava as an index of right-sided cardiac function. Am J Cardiol. 1984;53(4): 579-585.

9. Mintz GS, Kotler MN, Parry WR, Iskandrian AS, Kane SA. Reat-time inferior vena caval ultrasonography: normal and abnormal findings and its use in assessing right-heart function. Circulation. 1981;64(5):1018-1025.

10. Yanagawa Y, Nishi K, Sakamoto T, Okada Y. Early diagnosis of hypovolemic shock by sonographic measurement of inferior vena cava in trauma patients. J Trauma. 2005;58(4):825-829.

11. Lyon M, Blaivas M, Brannam L. Sonographic measurement of the inferior vena cava as a marker of blood loss. Am J Emerg Med. 2005;23(1):45-50.

12. Sefidbakht S, Assadsangabi R, Abbasi HR, Nabavizadeh A. Sonographic measurement of the inferior vena cava as a predictor of shock in trauma patients. Emerg Radiol. 2007;14(3):181-185.

13. Kusaba T, Yamaguchi K, Oda H, Harada T. Echography of inferior vena cava for estimating fluid removed from patients undergoing hemodialysis. Nihon Jinzo Gakkai Shi. 1994;36(8):914-920.

14. Rudski LG, Lai WW, Afilalo J, et al. Guidelines for the echocardiographic assessment of the right heart in adults: a report from the American Society of Echocardiography endorsed by the European Association of Echocardiography, a registered branch of the European Society of Cardiology, and the Canadian Society of Echocardiography. J Am Soc Echocardiogr. 2010;23(7):685-713; quiz 786.

15. Schefold JC, Storm C, Bercker S, et al. Inferior vena cava diameter correlates with invasive hemodynamic measures in mechanically ventilated intensive care unit patients with sepsis. J Emerg Med. 2010;38(5):632-637. 
16. Randazzo MR, Snoey ER, Levitt MA, Binder K. Accuracy of emergency physician assessment of left ventricular ejection fraction and central venous pressure using echocardiography. Acad Emerg Med. 2003;10(9): 973-977.

17. Brennan JM, Blair JE, Goonewardena S, et al. Reappraisal of the use of inferior vena cava for estimating right atrial pressure. $J$ Am Soc Echocardiogr. 2007;20(7):857-861.
18. Rivers E, Nguyen B, Havstad S, et al; Early Goal-Directed Therapy Collaborative Group. Early goal-directed therapy in the treatment of severe sepsis and septic shock. N Engl J Med. 2001;345(19): 1368-1377.

\section{Publish your work in this journal}

Open Access Emergency Medicine is an international, peer-reviewed, open access journal publishing original research, reports, editorials, reviews and commentaries on all aspects of emergency medicine. The manuscript management system is completely online and includes a very quick and fair peer-review system, which is all easy to use.
Visit http://www.dovepress.com/testimonials.php to read real quotes from published authors. 